\title{
Placental Disc Thickness
}

National Cancer Institute

\section{Source}

National Cancer Institute. Placental Disc Thickness. NCI Thesaurus. Code C117342.

The measured or estimated height of the placental disc perpendicular to the chorionic and basal plates. 